Extending the classification to probable besides defined, 38/60 (63.3\%) met Bohan and Peter criteria and 51/60 (85\%) met EULAR/ACR criteria.

Table 1 shows percentage of patients meeting each criteria set by clinical subtype.

Patients with available muscle biopsy $(n=14)$ were subanalyzed: $11 / 14(78.6 \%)$ muscle biopsy were compatible with IIM (3 not compatible, but previously treated). DM $3 / 11$ (28.6\%), ASS 1/11 (7.14\%) y CTD-OM 7/11 (50\%). 7/11 (50\%) met Bohan and Peter criteria, 8/11 (78.6\%) met Tanimoto criteria and 8/11 met EULAR/ACR criteria. If extended to probable cases, $11 / 11(100 \%)$ met Bohan and Peter criteria and 10/11 (91\%) EULAR/ACR criteria

Table 1 Fullfillment of different criteria set by IIM subtype

\begin{tabular}{|c|c|c|c|c|c|c|c|}
\hline & \multicolumn{3}{|c|}{ Bohan \& Peter (\%) } & \multirow{2}{*}{ Tanimoto (\% } & \multicolumn{3}{|c|}{ EULAR/ACR (\%) } \\
\hline & Probable & Definite & Total & & Probable & Definite & Total \\
\hline $\mathrm{DM}(\mathrm{n}=20)$ & 60 & 25 & 85 & 35 & 10 & 85 & 95 \\
\hline $\operatorname{CADM}(n=4)$ & 0 & 0 & 0 & 0 & 25 & 75 & 100 \\
\hline$P M(n=4)$ & 75 & 0 & 75 & 50 & 50 & 0 & 50 \\
\hline ASS $(n=10)$ & 50 & 10 & 60 & 80 & 30 & 70 & 100 \\
\hline CTD-OM (n=22) & 27,3 & 27,3 & 54,6 & $54,50 \%$ & 41 & 31,8 & 72,8 \\
\hline DM/CTD-OM (n=4) & 25 & 50 & 75 & 75 & 0 & 100 & 100 \\
\hline PM/CTD-OM (n=18) & 27,8 & 22,2 & 50 & 55,5 & 50 & 16,6 & 66,6 \\
\hline
\end{tabular}

Conclusions: EULAR/ACR 2017 performed especially well for DM, and were the only criteria set met by CADM patients.

PM patients failed to meet Bohan and Peter and EULAR/ACR criteria for definite disease, probably due to low availability of EMG and muscle biopsy. This was similar for pure PM and PM/CTD-OM.

Tanimoto criteria were the most sensitive for clasification of ASS patients, which could be explained because they consider arthritis among clinical features.

When considering probable and defined cases, EULAR/ACR criteria were highly sensible in this real world cohort

Disclosure of Interest: None declared

DOI: 10.1136/annrheumdis-2018-eular.6485

\section{THU0423 EPIDEMIOLOGY AND SURVIVAL OF SYSTEMIC SCLEROSIS-SYSTEMIC LUPUS ERYTHEMATOSUS OVERLAP SYNDROME}

S. Alharbi ${ }^{1,2,3,{ }^{*}, \text { Z. Ahmad }}{ }^{2}$, A. Bookman ${ }^{1}$, Z. Touma ${ }^{1,4}$, J. Sanchez-Guerrero ${ }^{1,2}$ N. Mitsakakis ${ }^{4,5}$, S.R. Johnson ${ }^{1,2,4}$. 'Toronto Scleroderma Program, Division of Rheumatology, Toronto Western Hospital, University Health Network; ${ }^{2}$ Division of Rheumatology, Mount Sinai Hospital, University of Toronto, Toronto, Canada; ${ }^{3}$ Taibah University, Medina, Saudi Arabia; ${ }^{4}$ Institute of Health Policy, Management and Evaluation; ${ }^{5}$ Toronto Health Economics and Technology Assessment Collaborative, University of Toronto, Toronto, Canada

Background: Systemic sclerosis (SSc) is an immune disorder characterised by vasculopathy and fibrosis that may overlap with another disease such as systemic lupus erythematous (SLE). Little is known about the epidemiology, clinical characteristics, and survival of SSc-SLE overlap (also called lupoderma). We evaluated the prevalence of SSc-SLE overlap syndrome, differences in SSc clinical characteristics and survival compared with SSc without SLE.

Methods: A cohort study was conducted including subjects who fulfilled the ACREULAR classification criteria for SSc and/or the ACR criteria for SLE. The primary outcome was the time from diagnosis to death from all causes. Survival was evaluated using Kaplan Meier curves and Cox Proportional Hazard models.

Results: We identified 1252 subjects (SSc $n=1166$, SSc-SLE $n=86$ ) with a SScSLE prevalence of $6.8 \%$. SSc-SLE were younger at diagnosis ( 37.9 years versus 47.9 years, $p<0.001)$, more frequently had lupus anticoagulant $(6 \%$ versus $0.3 \%$, $\mathrm{p}<0.001)$, anticardiolipin antibody $(6 \%$ versus $0.9 \%, \mathrm{p}<0.001)$, and pulmonary arterial hypertension (PAH) $(52 \%$ versus $31 \%, p<0.001)$. SSc-SLE less frequently had calcinosis $(13 \%$ versus $27 \%, p=0.007)$, telangiectasia $(49 \%$ versus $75 \%$, $p<0.001)$ and diffuse subtype $(12 \%$ versus $35 \%, p<0.001)$. There were no significant differences in the occurrence of renal crisis (7\% versus $7 \%)$, interstitial lung disease (40\% versus $34 \%$ ), and digital ulcers (38\% versus $32 \%$ ). SSc-SLE had better survival (median 26.1 versus 22.4 years), but this was not statistically significant ( $\log$ rank $\mathrm{p}=0.06$ ). Female sex and diffuse subtype attenuated survival differences between groups (Hazard Ratio $0.70,95 \% \mathrm{Cl} 0.45,1.11$ ).

Conclusions: SSc-SLE are younger at diagnosis, more frequently have $\mathrm{PAH}$, and less frequently have cutaneous manifestations of SSc. SSc-SLE patients should be monitored for pulmonary hypertension, interstitial lung disease, renal crisis and digital ulcers.

Disclosure of Interest: None declared

DOI: 10.1136/annrheumdis-2018-eular.1576

\section{THU0424 \\ ARTICULAR INVOLVEMENT IN SYSTEMIC SCLEROSIS: COMPARISON OF CLINICAL, RADIOGRAPHIC AND} SONOGRAPHIC FINDINGS

S. Thietart ${ }^{1}$, K. Louati ${ }^{2}$, E. Gaigneux ${ }^{2}$, M. Gatfosse ${ }^{1}$, S. Riviere ${ }^{1}$, J. Sellam ${ }^{2}$, F. Berenbaum ${ }^{2}$, O. Fain ${ }^{1}$, A. Mekinian ${ }^{1} .{ }^{1}$ Internal medecine; ${ }^{2}$ Rheumatology, Hôpital Saint Antoine, AP-HP, Université Paris 6, 75012 Paris, France., Paris, France

Background: Joint involvement in Systemic Sclerosis (SSc) is frequent and varied..$^{1,2}$

Objectives: We study US synovitis and its correlation with clinical synovitis, radiological erosions and organ involvement.

Methods: In a prospective cohort of SSc patients, tender and swollen joint counts, DAS28-CRP, hand US sonographies, X-ray hand views, as well as respiratory, cardiac, cutaneous and renal characteristics were assessed.

Results: 54 patients were included with a median age of 59 years, ${ }^{27-81} 45$ women (83\%), with a diffuse cutaneous subtype in 13 patients $(24 \%) .23$ patients $(52 \%)$ presented with arthralgia, 9 had clinical synovitis (16\%) and DAS28-CRP of 3.7 (2.98-5.90). US sonography (34 patients) found at least one synovitis in 23 patients: 14 patients with grade $1(66 \%), 6$ patients grade $2(29 \%), 1$ patient grade $3(5 \%)$, with a positive power Doppler signal in one case (3\%). Among the patients having US-synovitis, 4 had clinical synovitis (17\%), and 4 had X-ray erosions (17\%). Radiological erosions were present in 8 patients (15\%), without any correlation with clinical or US synovitis. Articular involvement (defined as clinical synovitis, US-synovitis and/or articular erosions) were found more frequently in limited SSc $(n=28,72 \%)$ than in diffuse SSc $(n=4,31 \%)(p<0.001)$, with a more frequent positivity of anti-centromere antibodies ( $n=23,60 \%$ versus $n=3,20 \%$ ). No correlation was found with disease severity or other organ impairment.

Conclusions: US synovitis were found more frequently than clinical synovitis, which are merely active, and did not correlate with articular destruction.

REFERENCES:

[1] Avouac J, Walker U, Tyndall A, Kahan A, Matucci-Cerinic M, Allanore Y, et al. Characteristics of joint involvement and relationships with systemic inflammation in systemic sclerosis: results from the EULAR Scleroderma Trial and Research Group (EUSTAR) database. J Rheumatol. 2010 July;37(7):1488-501.

[2] Elhai M, Guerini H, Bazeli R, Avouac J, Freire V, Drapé J-L, et al. Ultrasonographic hand features in systemic sclerosis and correlates with clinical biologic, and radiographic findings. Arthritis Care Res (Hoboken). août 2012;64(8):1244-9.

Disclosure of Interest: None declared

DOI: 10.1136/annrheumdis-2018-eular.7217

\section{THU0425 18F-FLUORODEOXYGLUCOSE POSITRON EMISSION TOMOGRAPHY/COMPUTED TOMOGRAPHY AND LUNG} INVOLVEMENT IN SYSTEMIC SCLEROSIS

S. Bellando Randone ${ }^{1}$, L. Tartarelli ${ }^{2}$, E. Cavigli ${ }^{3}$, L. Tofani ${ }^{4}$, C. Bruni ${ }^{1}$, G. Lepri ${ }^{1}$ J. Blagojevic ${ }^{1}$, A. Moggi Pignone ${ }^{5}$, C. Mihai ${ }^{6}$, J. Avouac $^{7}$, A. Passeri², M.T. De Cristofaro $^{2}$, O. Distler ${ }^{6}$, Y. Allanore ${ }^{7}$, S. Guiducci ${ }^{1}$, M. Matucci Cerinic ${ }^{1}$. ${ }^{1}$ Dept of Experimental and Clinical Medicine, University of Florence, Italy and Dept of Geriatric Medicine, Div Rheumatology AOUC, ${ }^{2}$ Department of Biomedical, Experimental and Clinical Sciences, "Mario Serio", Nuclear Medicine Unit, University of Florence; ${ }^{3}$ Department of Radiodiagnostic and Emergency, Careggi University Hospital, Florence; ${ }^{4}$ Department of Neurosciences, Psychology, Drug Research and Child Health, University of Florence, Florence; ${ }^{5}$ Internal Medicine of Careggi University Hospital, Florence, Italy; ${ }^{6}$ Department of Rheumatology, University Hospital Zurich, Zurich, Switzerland; ${ }^{7}$ Department of Rheumatology A, Cochin Hospital, INSERM U1016, Paris Descartes University, Paris, France

Background: At early stages, SSc lung involvement is characterised by Ground Glass Opacities (GGO) at High Resolution Computed Tomography (HRCT). How ever, HRCT scan is not able to provide functional information and to discriminate between an "active inflammatory" and an "established fibrotic" GGO. 18FluoroDeoxy-d-Glucose (18F-FDG) Positron Emission Tomography/Computed Tomog raphy $(\mathrm{PET} / \mathrm{CT})$ is able to detect metabolic activity picking up inflammation and provides both morphologic and metabolic data.

Objectives: The aim of this study was to evaluate, if $18 \mathrm{~F}-\mathrm{FDG}$ PET/CT scan may identify the inflammatory component of GGO in SSc interstitial lung disease.

Methods: Seven patients with SSc (1 male and 6 females; mean age 59.56 y $\pm 9.15 \mathrm{SD}$; median disease duration 5 years, ${ }^{2-11}$ who underwent $18 \mathrm{~F}-\mathrm{FDG}$ PET/ CT scan because of cancer screening, were retrospectively analysed. HRCT 